\title{
Proton beam therapy for cancer
}

\author{
Derek S. Tsang MD MSc, Samir Patel MD
}

Cite as: CMAJ 2019 June 17;191:E664-6. doi: 10.1503/cmaj.190008

$\mathbf{R}$ adiotherapy is a central modality for treatment of cancer in one-third of cancer patients. It is designed to target tumour cells and damage their DNA, while using advanced shaping techniques such as beam modulation to avoid normal tissues. Radiotherapy in Canada is delivered using photon (x-ray) irradiation, which is slowly attenuated by human tissue. With photon radiotherapy, some dose will pass through the entire depth of the patient, thereby unnecessarily irradiating normal body tissues.

Children and young adults are exquisitely sensitive to radiotherapy, and minimizing the dose to normal tissues is of paramount importance. Among those who are cured of cancer, many end up with long-term adverse effects of therapy, including learning and memory problems, hearing loss, growth deformities, infertility, treatment-related secondary cancers and increased cardiovascular morbidity. Treatment protocols that decrease exposure to radiotherapy are associated with reduced risks of late mortality. ${ }^{1}$ Proton beam therapy is a new way to deliver radiation that addresses many of these concerns.

\section{What is proton beam therapy?}

Proton beam therapy (proton therapy) reduces the volume of normal tissues that receive unnecessary radiation during cancer treatment. The physical dose distribution produced by proton beams is superior relative to photon radiotherapy because of the reduced "exit dose" to normal tissues (Figure 1). High-energy protons shed some of the energy they carry as they pass through tissue and then deposit the majority at the end of their range, precisely within a tumour target.

\section{How is proton therapy delivered?}

Whereas photon radiotherapy is delivered from a standalone linear accelerator that fits within a single storey with a footprint of about $75 \mathrm{~m}^{2}$, proton therapy units require at least 3 storeys $(>8.4 \mathrm{~m}$ in height) with a footprint ranging from $111 \mathrm{~m}^{2}$ (single compact treatment unit) to $2000 \mathrm{~m}^{2}$ (4 full gantry treatment units) to accommodate the treatment gantry, cyclotron and supporting equipment. As with photon radiotherapy, most proton treatments are delivered over a fractionated course of therapy up to 7 weeks long.

As of April 2019, there were 81 operating proton beam therapy centres worldwide, 41 under construction and 20 centres

\section{KEY POINTS}

- Proton beam therapy can effectively treat cancer while reducing adverse effects by minimizing the volume of normal tissues exposed to radiation.

- Proton therapy is most useful for pediatric cancers, as well as many head-and-neck and brain tumours.

- Canada is the only G8 country without a clinical proton facility; patients must travel to the United States for treatment that may last 6-8 weeks.

- Provinces may provide funding to cover costs of treatment, but access to proton therapy remains challenging because of nonmedical costs and logistical difficulties of travel.

in the planning phase. ${ }^{2}$ Canada is the only G8 country without a hospital-based proton beam facility in operation or under construction. The United States has 31 facilities in operation. China and South Korea have been treating patients since 2004 and 2007, respectively. The United Kingdom, Netherlands and Austria all started treating patients with proton therapy in 2018, and India and Denmark each opened a facility in early 2019.

Presently, Canadian patients, many of whom are young children, must travel to the US for proton therapy. Many provinces do not cover nonmedical expenses, such as travel, accommodation or food. Patients, often young children, must also endure the psychosocial grief of being away from their family and friends, often for 6-8 weeks.

\section{Who is eligible for proton therapy?}

Alberta Health Services released a guideline in 2014 describing accepted indications for proton therapy, including base-of-skull tumours (chordoma, chondrosarcoma), intraocular melanoma, most pediatric tumours and some adult central nervous system tumours. ${ }^{3}$ A similar review in 2014 by Cancer Care Ontario's Proton Advisory Group supported its use for pediatric cancers and some head-and-neck and base-of-skull tumours. ${ }^{4}$ The American Society for Radiation Oncology has a model policy that supports insurance coverage for proton therapy in patients with ocular tumours, base-of-skull cancer, hepatocellular carcinoma, pediatric cancers, central nervous system tumours, advanced headand-neck cancer, retroperitoneal sarcoma and those with a 


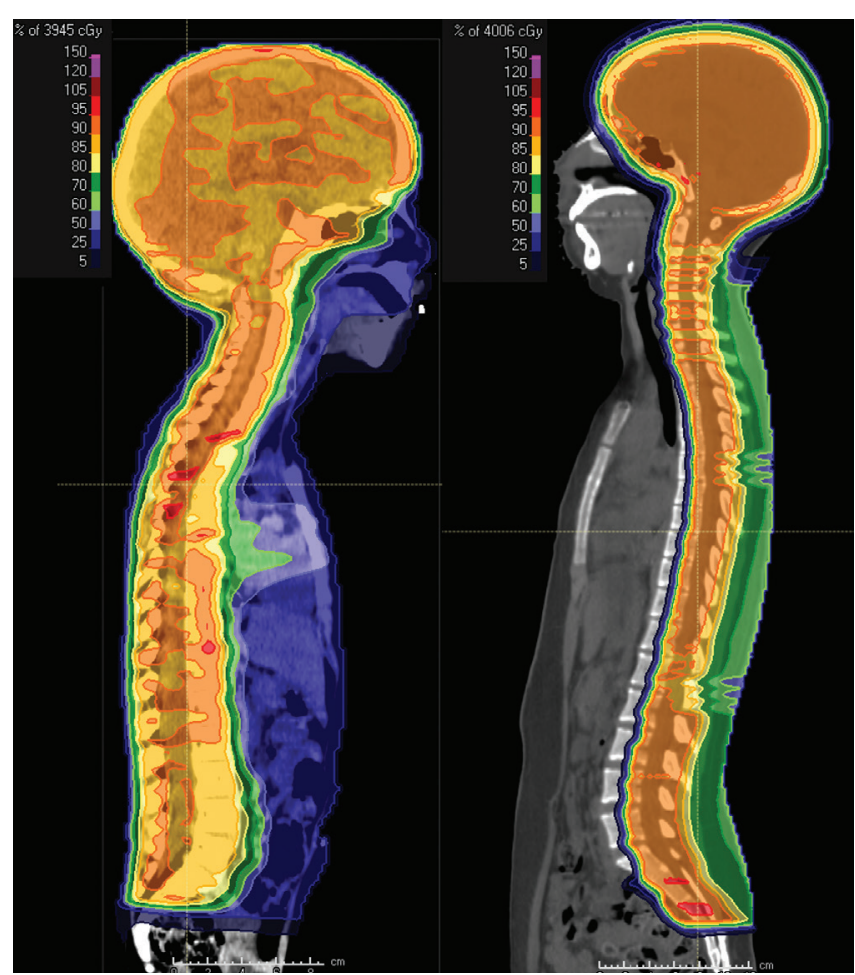

Figure 1: Midline sagittal computed tomography images of (A) a 4-year-old child with a malignant brain tumour, treated with photon craniospinal irradiation and (B) a 16-year-old child with a malignant brain tumour, treated with proton craniospinal irradiation. The coloured portions denote regions that are receiving at least $5 \%$ of the prescribed radiation dose of 3600 cGy. Regions in dark blue and light blue are receiving $25 \%$ and $50 \%$ of the prescribed dose, respectively. Regions in light green, dark green, yellow, orange or red are receiving $60 \%, 70 \%, 85 \%, 90 \%$ and $95 \%$ of the prescribed dose, respectively. Note the reduction in dose exposure to normal tissues in the face, neck and thyroid, heart, liver, and pelvis with proton therapy. This comes at the small cost of increased entrance and skin dose.

genetic predisposition to increased radiosensitivity, as well as for selected cases requiring re-irradiation. ${ }^{5}$

There is no national database that tracks all patients treated with proton therapy in Canada. In Canada, the Public Health Agency of Canada and the Cancer in Young People in Canada program (www.c17.ca/index.php?cID=70) collect data on youth younger than 15 years with cancer; an aggregate data query showed that at least 69 patients with non-leukemia cancer were treated with proton therapy between January 2001 and November 2018 (about $2 \%$ of the 3483 patients who received radiotherapy). This is lower than in the US, where use of the treatment grew from $0.3 \%$ of children treated in 2002 to $9.7 \%$ in $2012 .{ }^{6}$

\section{What are the harms?}

Early reports found a potentially increased risk of brainstem necrosis in patients treated with proton therapy for brain tumours. This may have been due to the increase in relative biological effectiveness at the end of a proton beam. The risk of brainstem toxicity can be successfully mitigated with careful radiation planning and adherence to dose guidelines to protect normal tissues. ${ }^{7}$
A recent randomized controlled trial comparing proton therapy with photon radiotherapy in lung cancer found no difference in local tumour failure, but the trial failed to meet its primary end point of reducing radiation pneumonitis. ${ }^{8} \mathrm{~A}$ secondary analysis found that patients treated with proton therapy who were enrolled early in the study had a nearly $25 \%$ risk of pneumonitis, in contrast to those enrolled late in the study, who did not develop any pneumonitis ( $p=0.01$ ), indicating a learning curve with the delivery of proton therapy. ${ }^{8}$

\section{What is the evidence so far?}

Because of reduction in irradiated normal tissue, proton therapy is expected to reduce the incidence of secondary, radiationinduced malignant neoplasms (adjusted hazard ratio 0.52, 95\% confidence interval 0.32-0.85)..$^{9,10}$

Proton therapy is a cost-effective treatment, especially for pediatric brain tumours (incremental cost-effectiveness ratio US\$21716-\$26419 per quality-adjusted life year [QALY], depending on the study) and some head-and-neck cancers (incremental cost-effectiveness ratio US\$4254-\$143229 per QALY, depending on the study and radiation techniques), ${ }^{11}$ below a commonly accepted willingness-to-pay threshold of $\$ 150000$ per QALY.

Although the dosimetric benefits of proton therapy are clear, evidence in support of the treatment is limited by a lack of highquality, randomized studies. The Canadian Agency for Drugs and Technologies in Health issued a health technology assessment of proton therapy in August 2017.12 This report concluded that proton therapy provides similar cancer-control efficacy to photon radiotherapy and that a single unit in Canada is expected to save health care payers $\$ 12.9$ million over a 10 -year time horizon, as compared with continuing out-of-country referrals.

\section{What can be expected in the future?}

With increasing awareness of proton therapy as a treatment for children and selected young adults with cancer, more patients will likely be sent to the US for treatment over time. In Ontario and Alberta, preferred provider arrangements are being negotiated that will formalize care pathways for patients needing this treatment in the US. A business consortium in Quebec recently announced plans for a private facility on the Island of Montreal; ${ }^{13}$ details of how publicly funded patients from the rest of Canada can access this resource are not yet determined. Until proton therapy is broadly available across the country, challenges in accessing this advanced radiotherapy modality will likely persist.

\section{References}

1. Armstrong GT, Chen Y, Yasui Y, et al. Reduction in late mortality among 5-year survivors of childhood cancer. N Engl J Med 2016;374:833-42.

2. Particle Therapy Co-Operative Group [main page]. Available: www.ptcog.ch (accessed 2019 May 23).

3. Patel S, Kostaras X, Parliament M, et al. Recommendations for the referral of patients for proton-beam therapy, an Alberta Health Services report: A model for Canada? Curr Oncol 2014;21:251-62.

4. Access to proton therapy for cancer patients in Ontario. A report by Cancer Care Ontario's Proton Advisory Group. Toronto: Cancer Care Ontario; 2014. 
5. ASTRO Model Policies: Proton Beam Therapy (PBT). Arlington (VA): American Society for Radiation Oncology; 2017.

6. Waddle MR, Sio TT, Van Houten HK, et al. Photon and proton radiation therapy utilization in a population of more than 100 million commercially insured patients. Int J Radiat Oncol Biol Phys 2017;99:1078-82.

7. Haas-Kogan D, Indelicato D, Paganetti H, et al. National Cancer Institute Workshop on Proton Therapy for Children: considerations regarding brainstem injury. Int J Radiat Oncol Biol Phys 2018;101:152-68.

8. Liao Z, Lee JJ, Komaki R, et al. Bayesian adaptive randomization trial of passive scattering proton therapy and intensity-modulated photon radiotherapy for locally advanced non-small-cell lung cancer. J Clin Oncol 2018; 36:1813-22.

9. Eaton BR, MacDonald SM, Yock TI, et al. Secondary malignancy risk following proton radiation therapy. Front Oncol 2015;5:261.
10. Chung CS, Yock TI, Nelson K, et al. Incidence of second malignancies among patients treated with proton versus photon radiation. Int J Radiat Oncol Biol Phys 2013;87:46-52.

11. Verma V, Mishra MV, Mehta MP. A systematic review of the cost and costeffectiveness studies of proton radiotherapy. Cancer 2016;122:1483-501.

12. Proton beam therapy for the treatment of cancer in children and adults: $a$ health technology assessment. Issue No 145. Ottawa: Canadian Agency for Drugs and Technologies in Health; 2017 Aug. 15.

13. CDL Laboratories Inc. Quebec-based CDL Laboratories to build $\$ 70 \mathrm{M}$ state-ofthe-art proton therapy cancer centre in Montreal, the first of its kind in Canada. Toronto: Canada News Wire (CNW) Group Ltd.; 2018 Aug. 22. Available: www. newswire.ca/news-releases/quebec-based-cdl-laboratories-to-build-70-m-state -of-the-art-proton-therapy-cancer-centre-in-montreal-the-first-of-its-kind-in -canada-691462591.html (accessed 2018 Aug. 22).
Competing interests: The Radiation Medicine Program at the Princess Margaret Cancer Centre received unrestricted educational funds for a symposium in November 2018 from Varian Medical Systems, Mevion Medical Systems, Hitachi, RaySearch Laboratories, IBA and ProTom. Derek Tsang was a co-chair of the symposium and has not received any financial benefits, salaries, honoraria or stipends from any commercial entity related to this work. Samir Patel was an invited faculty speaker at the symposium and has not received any financial benefits, salaries, honoraria or stipends from any commercial entity related to this work.

This article has been peer reviewed.
Affiliations: Radiation Medicine Program (Tsang), Princess Margaret Cancer Centre, University Health Network; Division of Haematology/ Oncology (Tsang), Hospital for Sick Children, Toronto, Ont.; Divisions of Radiation Oncology and Pediatric Oncology (Patel), Cross Cancer Institute and Stollery Children's Hospital, University of Alberta, Edmonton, Alta.

Contributors: Derek Tsang conceived the idea for the work. Both authors analyzed and interpreted the data, wrote the manuscript, gave final approval of the version to be published and agreed to be accountable for all aspects of the work.
Acknowledgements: For the Cancer in Young People in Canada program aggregate data request, the authors gratefully acknowledge participating pediatric oncology centres, members of the Cancer in Young People in Canada management and steering committees, and the Pediatric Oncology Group of Ontario. Cancer in Young People in Canada is fully funded by the Public Health Agency of Canada.

Correspondence to: Derek Tsang, derek. tsang@rmp.uhn.ca 